\title{
Observation of ultralow-level Al impurities on a silicon surface by high-resolution grazing emission $x$-ray fluorescence excited by synchrotron radiation
}

\author{
A. Kubala-Kukuś, ${ }^{1}$ D. Banaś, ${ }^{1}$ W. Cao, ${ }^{2}$ J.-Cl. Dousse, ${ }^{2}$ J. Hoszowska, ${ }^{2}$ Y. Kayser, ${ }^{2}$ M. Pajek, ${ }^{1}$ M. Salomé, ${ }^{3}$ J. Susini, ${ }^{3}$ \\ J. Szlachetko, ${ }^{1,2}$ and M. Szlachetko ${ }^{2}$ \\ ${ }^{1}$ Institute of Physics, Jan Kochanowski University, 25-406 Kielce, Poland \\ ${ }^{2}$ Department of Physics, University of Fribourg, CH-1700 Fribourg, Switzerland \\ ${ }^{3}$ European Synchrotron Radiation Facility (ESRF), F-38043 Grenoble, France
}

\begin{abstract}
We demonstrate that ultralow-level Al impurities on a silicon surface can be measured by using the highresolution grazing emission $\mathrm{x}$-ray fluorescence (GEXRF) technique combined with synchrotron-radiation excitation. An Al-impurity level of about $10^{12}$ atoms $/ \mathrm{cm}^{2}$ was reached by observing the $\mathrm{Al} K \alpha$ x-ray fluorescence in the resonant Raman-scattering background-"free" regime by choosing an appropriate beam energy below the Si $K$ absorption edge. Present results show that by combining the GEXRF method with the vapor phase decomposition technique the $10^{7}$ atoms $/ \mathrm{cm}^{2}$ level can be reached for $\mathrm{Al}$ detection on silicon. Finally, we found that the high-resolution GEXRF technique is a sensitive tool to study the morphology of surface nanostructures.
\end{abstract}

Recent developments in nanoscience set new demands for the quality of materials, which can be fulfilled by developing techniques based on fundamentally new physical approaches. In fact, fast progress in Si-based microelectronic technology requires new techniques to control metallic impurities on a silicon surface at an ultralow level, well below the $10^{10}$ atoms $/ \mathrm{cm}^{2}$ limit, to fulfill an ongoing trend of miniaturization of electronic devices. These aspect also requires a nanoscale spatial resolution of new techniques to be sensitive for the studies of nanostructures.

At present, the application of the total reflection x-ray fluorescence (TXRF) technique, ${ }^{1}$ which uses the grazing incidence geometry to excite the x-ray fluorescence of studied materials by synchrotron-radiation $\mathrm{x}$ rays offers the best possibilities for measuring very low concentrations of metallic impurities on Si surface. The sensitivity of the TXRF method can be further enhanced in conjunction with the vapor phase deposition (VPD) preconcentration technique by collecting the impurities from the whole surface (see Ref. 2). Consequently, a combination of the TXRF/VPD techniques gives presently an access to measure the low- $Z$ impurities, and, in particular, $\mathrm{Al}$, on $\mathrm{Si}$ wafers at the level of $10^{9}$ atoms $/ \mathrm{cm}^{2} .^{3}$ Among X-ray-fluorescence-based analytical tools the grazing emission $\mathrm{x}$-ray fluorescence (GEXRF) technique ${ }^{4,5}$ has the potential of coping with the growing demands for detection of impurities on Si wafer surfaces at an ultralow level.

The beginning of the GEXRF technique goes back to the phenomenon of the total external reflection of $\mathrm{x}$ rays discovered by Compton. ${ }^{6}$ The analytical capabilities of the total reflection effect were recognized much later by Yoneda and Horiuchi $^{7}$ in their pioneering work on the applications of the total reflection $\mathrm{x}$-ray fluorescence. The idea to use the total reflection in the "inverse" mode for analytical applications was introduced by Becker, Golovchenko, and Patel. ${ }^{4}$ The principles of the GEXRF method were described in detail by Urbach and de Bokx in Ref. 5.

In this Brief Report we report on measurements of ultralow amounts of $\mathrm{Al}$ impurities on the surface of Si wafers by using the GEXRF technique in combination with highresolution X-ray detection and excitation by polarized photons from a synchrotron-radiation source. In the GEXRF technique, which is a kind of inverse of the TXRF method, the X-ray fluorescence is observed at angles smaller than the critical angles of the studied surface elemental impurities. This results in a substantial suppression of the $\mathrm{Si} K \alpha$ fluorescence emission (see Fig. 1), which is then limited to the sole evanescent $\mathrm{x}$-ray waves ${ }^{4}$ propagating along the surface and forming the $\mathrm{x}$-ray background for the fluorescence $\mathrm{x}$-ray emission from the impurities. As a consequence, the x-ray emission from the $\mathrm{Si}$ substrate is restricted to a very shallow

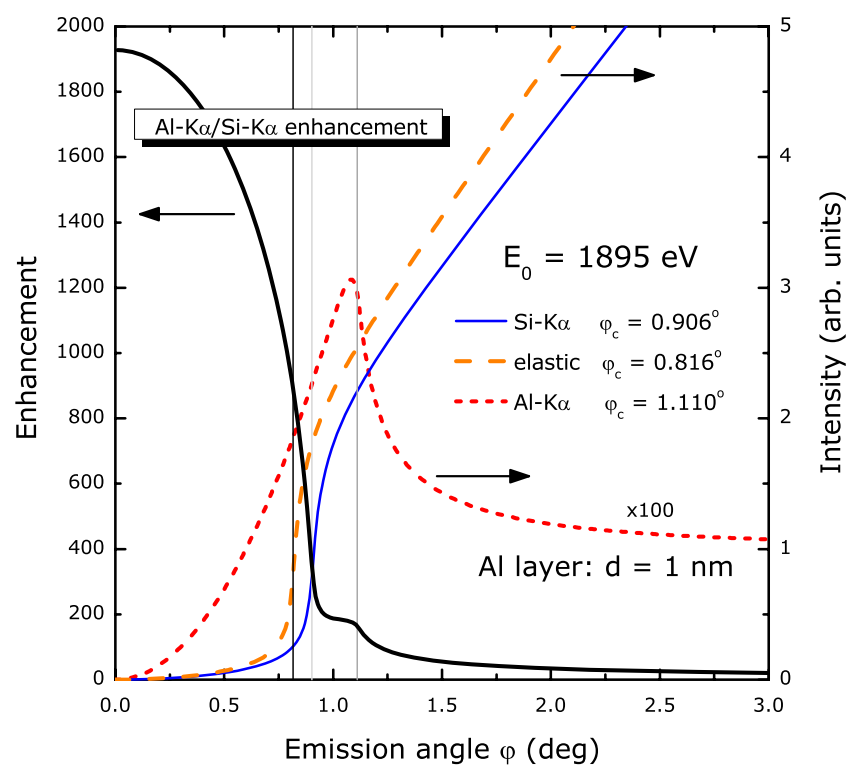

FIG. 1. (Color online) Calculated (Ref. 5) intensities of Al $K \alpha$ and Si $K \alpha$ fluorescence and elastically scattered photons of energy $E_{0}=1895 \mathrm{eV}$, as well as $\mathrm{Al} K \alpha / \mathrm{Si} K \alpha$ enhancement, plotted versus the emission angle for a 1-nm-thick Al layer on a Si wafer. Vertical lines indicate the corresponding critical angles. 
surface layer having a thickness of several nanometers. The basic features of the GEXRF method applied to the study of $\mathrm{Al}$ impurities in Si are illustrated in Fig. 1. As shown, below the critical angle, a three orders-of-magnitude enhancement of the $\mathrm{Al} K \alpha / \mathrm{Si} K \alpha$ intensity ratio is found for a $1-\mathrm{nm}$-thick Al layer on $\mathrm{Si}$.

The GEXRF measurements were performed at the beam line ID21 of the European Synchrotron-Radiation Facility (ESRF), in Grenoble, France, by means of high-resolution $\mathrm{X}$-ray spectroscopy, using a von Hamos Bragg-type curved crystal spectrometer ${ }^{8}$ having an energy resolution of about $0.9 \mathrm{eV}$. The photon beam delivered by the undulator was monochromatized $(\Delta E \sim 6 \mathrm{eV})$ by means of two $20 \AA$ $\mathrm{Ni} / \mathrm{B}_{4} \mathrm{C}$ multilayers. Higher-energy photons corresponding to upper harmonics were suppressed with a Ni mirror. Using a circular $1 \mathrm{~mm}$ in diameter pinhole located between the $\mathrm{Ni}$ mirror and the beam port of the spectrometer chamber, about $10^{11}$ photons/s were obtained on the samples. The latter consisted of 0.3 -mm-thick standard silicon $\langle 100\rangle$ wafers produced for microelectronic applications. The angle between the surface of the wafers and the incoming photon beam was $39^{\circ}$. For the present experiment, the von Hamos spectrometer was equipped with a $10 \mathrm{~cm}$ high $\times 5 \mathrm{~cm}$ wide $\times 0.5 \mathrm{~mm}$ thick ammonium dihydrogen phosphate (101) crystal $(2 d=10.642 \AA)$ bent cylindrically to a radius of 25.4 $\mathrm{cm}$. For the detection of the diffracted photons a $26.8 \mathrm{~mm}$ long $\times 8 \mathrm{~mm}$ high back-illuminated chargecoupled device (CCD) camera with a pixel resolution of $20 \mu \mathrm{m}$ was employed. In the high-resolution GEXRF method no narrow slit is needed between the sample and the crystal since for small emission angles the source of the fluorescence photons is seen by the crystal as a linelike source. A further advantage provided by the high-resolution setup is that a substantial diminution of the x-ray background resulting from the elastic scattering of incoming photons by the sample can be obtained if the photons are linearly polarized in the dispersion plane of the spectrometer and the Bragg angle is close to $45^{\circ}$. As these two conditions were fulfilled in our experiment, almost no elastic-scattering background was observed in our high-resolution $x$-ray spectra. The grazing $\mathrm{X}$-ray emission observation angle $\varphi$ should lie below the critical angle $\varphi_{c}=0.906^{\circ}$ corresponding to the Si $K \alpha$ fluorescence in order to minimize the $\mathrm{x}$-ray background from the bulk silicon and close to the critical angle $\varphi_{c}=1.110^{\circ}$ for which the maximum intensity of the $\mathrm{Al} K \alpha$ fluorescence from the thin surface impurity layer is expected (see Fig. 1). For these reasons an observation angle $\varphi=0.6^{\circ}$ was chosen as the best compromise between these two conditions. The variation in the $\mathrm{Al} K \alpha$ to $\mathrm{Si} K \alpha$ intensity ratio as a function of the emission angle is shown in Fig. 1.

The x-ray emission from the bulk silicon can be further diminished at the grazing emission condition $\left(\varphi<\varphi_{c}\right)$ by choosing an appropriate incident photon beam energy. First, while being higher than the $\mathrm{Al} K$ absorption edge $(1560 \mathrm{eV})$, the photon beam energy should be lower than the Si $K$ absorption edge $(1840 \mathrm{eV})$ to avoid the strong fluorescence emission of the Si $K \alpha$ line from bulk silicon. However, for photon beam energies just below the Si $K$ absorption edge the resonant Raman-scattering (RRS) process $^{3,9}$ appears, giving the main contribution to the $\mathrm{X}$-ray background interfering

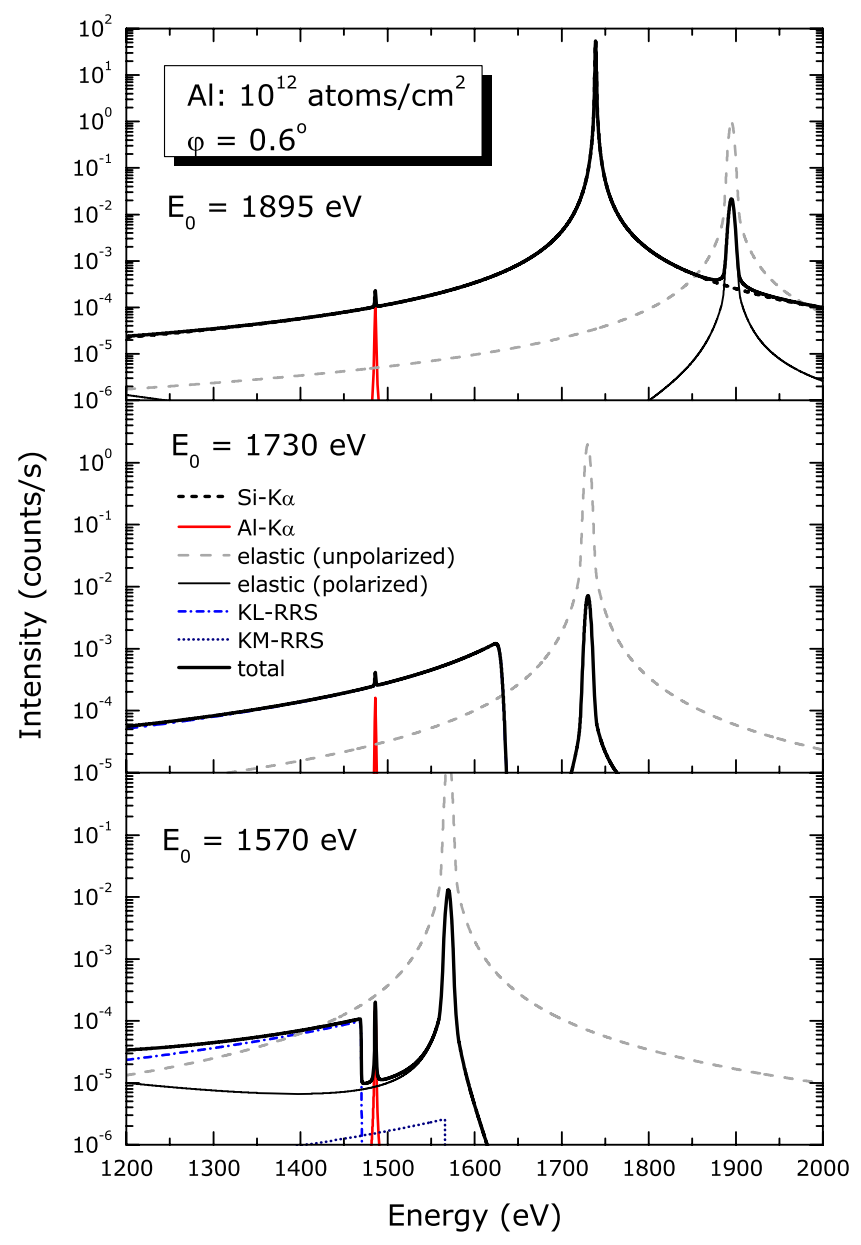

FIG. 2. (Color online) Calculated GEXRF x-ray spectra showing the $\mathrm{Al} K \alpha$ fluorescence line, the $\mathrm{x}$-ray background from $\mathrm{KL}$ RRS and KM-RRS processes and the elastic scattering of incident photons with energies of $1895 \mathrm{eV}$ (top), $1730 \mathrm{eV}$ (middle), and $1570 \mathrm{eV}$ (bottom). The Si $K \alpha$ fluorescence line appears only for incident photon energy above the Si $K$ absorption edge.

with the $\mathrm{Al} K \alpha$ fluorescence. In fact, the KL-RRS and KMRRS processes, with KL or KM denoting the initial and final electronic state involved, set the lower detection limit for observation of low-level amounts of aluminum on silicon. In order to investigate the RRS process in more details we have measured recently ${ }^{10}$ the high-resolution RRS $\mathrm{x}$-ray spectra for $\mathrm{Si}$ below the $K$ edge. Guided by these results, for the present GEXRF measurements we have chosen an X-ray photon beam energy of $1570 \mathrm{eV}$. For this beam energy, just above the $\mathrm{Al} K$ absorption edge, the high-energy cutoff of the Si KL-RRS spectrum lies at about $1470 \mathrm{eV}$, i.e., below the Al $K \alpha$ fluorescence line (see Fig. 2) so that the GEXRF measurements become nearly free of Raman-scattering background, with only the almost two orders of magnitude weaker $(3 \%)$ KM-RRS process contributing to the Raman $\mathrm{X}$-ray background. For the two other incident photon beamenergy regimes shown in Fig. 2, namely, $E_{0}=1730 \mathrm{eV}$ and $E_{0}=1895 \mathrm{eV}$, the x-ray background was found to be mainly due to the KL-RRS structure and the Lorentzian profile of the Si $K \alpha$ fluorescence line, respectively. Actually, as expected, the best signal-to-background ratio was observed for 


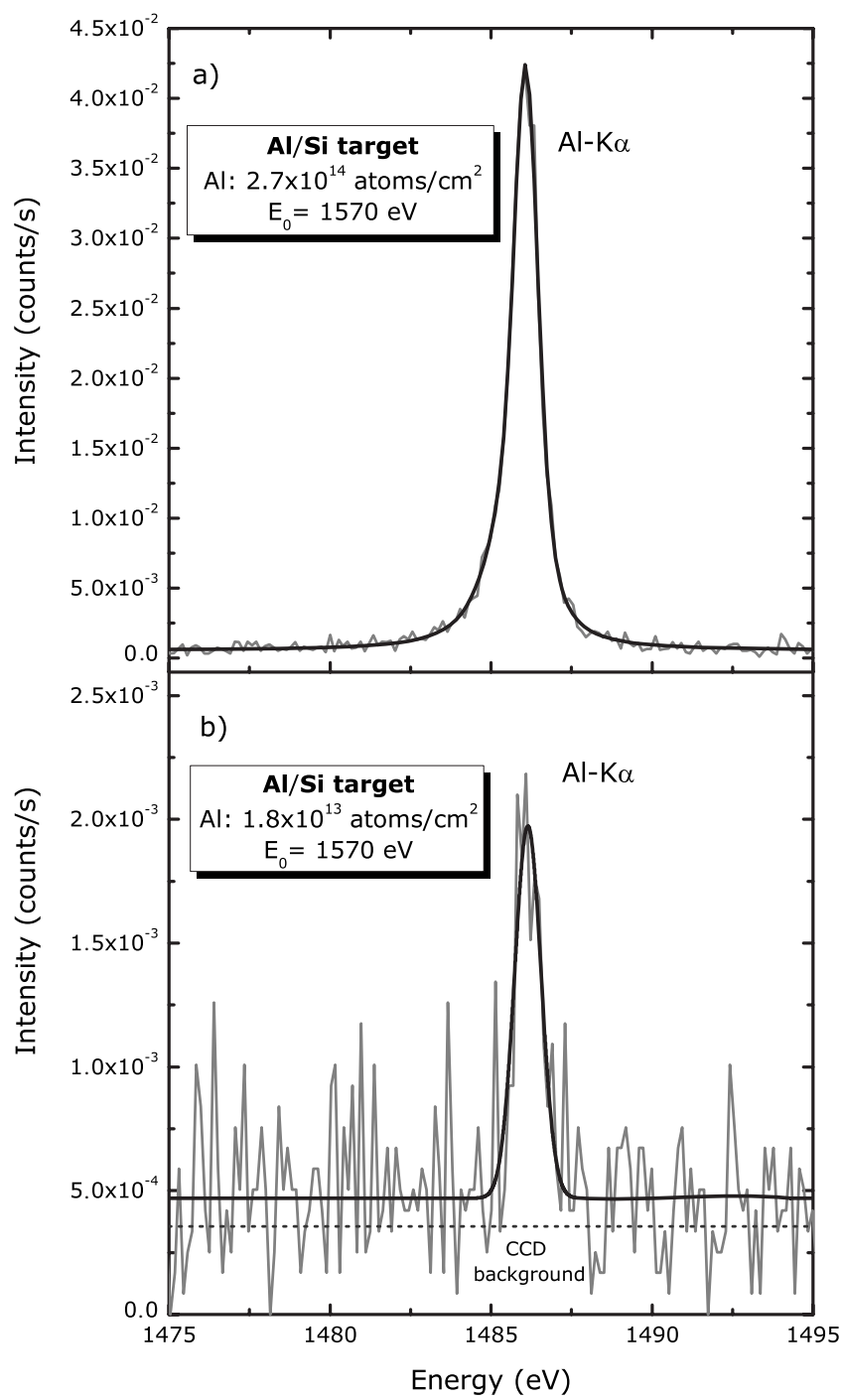

FIG. 3. High-resolution Al $K \alpha$ fluorescence x-ray lines measured at a grazing emission angle of $\varphi=0.6^{\circ}$ for photon beam energy of $1570 \mathrm{eV}$ for: (a) an intentionally contaminated with 2.7 $\times 10^{14} \mathrm{Al}$ atoms $/ \mathrm{cm}^{2} \mathrm{Si}$ wafer and (b) a blank $\mathrm{Si}$ wafer with a $1.8 \times 10^{13} \mathrm{Al}$ atoms $/ \mathrm{cm}^{2}$ surface contamination.

the photon beam energy $E_{0}=1570 \mathrm{eV}$. It is important to emphasize that the use of high resolution for the X-ray detection in the GEXRF technique was a crucial point in the present experiments to separate the $\mathrm{Al} K \alpha$ line from the interfering RRS structures.

The measured high-resolution $\mathrm{Al} K \alpha$ fluorescence x-ray line from a $\mathrm{Si}$ wafer intentionally contaminated with $\mathrm{Al}$ atoms at the level of about $3 \times 10^{14}$ atoms $/ \mathrm{cm}^{2}$ using an $\mathrm{Al}\left(\mathrm{NO}_{3}\right)_{3}$ in $\mathrm{HNO}_{3}$ solution is shown in Fig. 3. For comparison, the same $\mathrm{x}$-ray line but from aluminum impurities in a blank $\mathrm{Si}$ wafer is also shown. It was found that the lowintensity $\left(\sim 2 \times 10^{-5}\right.$ counts/s) X-ray background observed in the high-resolution GEXRF x-ray spectra was mainly due to cosmic radiation and natural radioactivity events in the CCD detector and was not related to the incident photon beam. It can thus be concluded that the sensitivity of the present high-resolution GEXRF measurements of Al impuri-

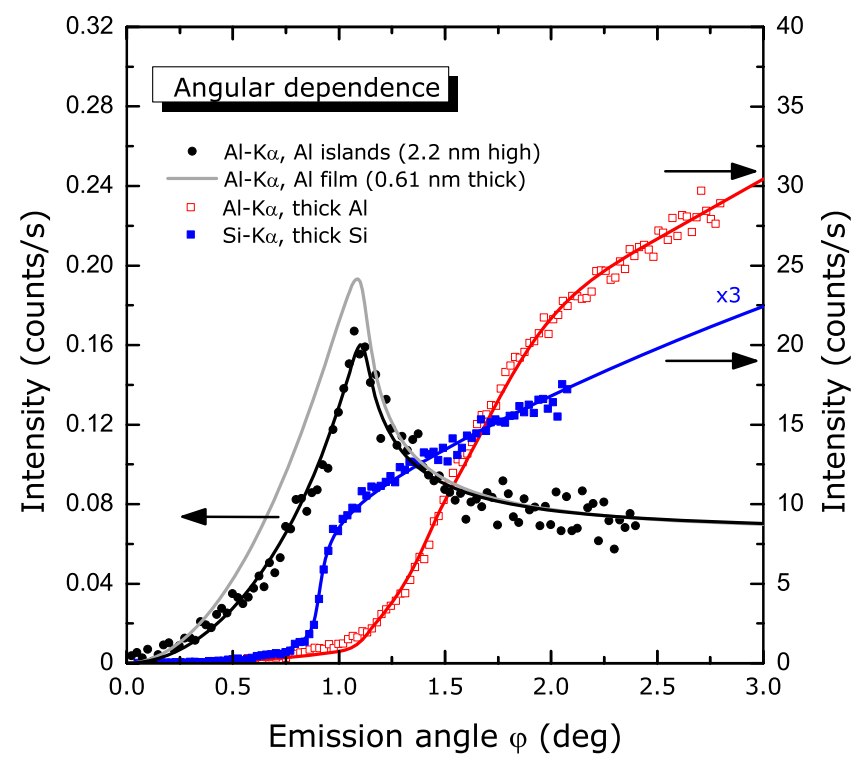

FIG. 4. (Color online) Measured intensities of the $\mathrm{Al} K \alpha$ fluorescence line excited by $1570 \mathrm{eV}$ photons versus the grazing emission angle for a thin $\mathrm{Al}$ layer on a $\mathrm{Si}$ wafer and a thick $\mathrm{Al}$ sample. The angular dependence of the $K \alpha$ fluorescence line of a thick Si sample bombarded by $2000 \mathrm{eV}$ photons is also shown. The solid lines are the results of calculations (Ref. 5) performed for filmlike (0.61-nm-thick) and islandlike (2.2-nm-high) Al layers (left scale), and thick $\mathrm{Al}_{2} \mathrm{O}_{3} / \mathrm{Al}$ and $\mathrm{Si}$ samples (right scale).

ties on $\mathrm{Si}$ wafers was mainly limited by the natural x-ray background.

In order to convert the measured intensities of the $\mathrm{Al} K \alpha$ fluorescence line into surface concentrations of Al impurities, separate GEXRF measurements were performed with the same experimental conditions but using a thick aluminum target and a thin Al layer (about $1 \mathrm{~nm}$ thick) evaporated on a $\mathrm{Si}$ substrate. In these measurements the x-ray fluorescence yields were determined as a function of the x-ray emission angle and the results were compared with calculated GEXRF predictions following Ref. 5. Similar measurements were also performed with a thick silicon sample for the calibration of the $\mathrm{x}$-ray emission angle, using a beam energy of $2000 \mathrm{eV}$. Results of the Al layer, thick Al, and thick Si measurements are shown in Fig. 4. For the thick Al, the measured angular dependence could be only well reproduced if a 15-nm-thick layer of $\mathrm{Al}_{2} \mathrm{O}_{3}$ was assumed on the surface of the sample. This assumption was confirmed by an independent $\mathrm{x}$-ray absorption near-edge structure measurement. By comparing the fluorescence intensities of the thin and thick Al samples at an emission angle $\varphi=2.0^{\circ}$, a thickness of 0.61 $\mathrm{nm}$ was found for the Al layer. Note that for the thin Al layer no effect of $\mathrm{Al}$ oxidation is expected according to the GEXRF calculations. ${ }^{5}$ However, as shown in Fig. 4, the angular dependence obtained for this sample could not be reproduced satisfactorily, particularly below the critical angle, by calculations assuming an uniform filmlike layer having the above-mentioned thickness of $0.61 \mathrm{~nm}$. In contrast to that, it was found that the measured intensities could be well reproduced assuming an islandlike instead of filmlike morphology for the Al evaporated onto the Si surface. Actually, 
the formation of islands is a well-known Volmer-Weber process of thin-film growth, which is responsible for the formation of quite regular nanometer-scale islands, ${ }^{11}$ in particular, occurring in Al evaporation on silicon. ${ }^{12}$ Guided by these observations, we have found that the measured angular dependence for the $0.61 \mathrm{~nm} \mathrm{Al}$ "layer" on $\mathrm{Si}$ was best fitted assuming Al islands with an average height of $2.2 \mathrm{~nm}$, which is in qualitative agreement with the results of Ref. 12. This result demonstrates a sensitivity of the developed highresolution GEXRF technique for the morphology of the surface nanostructures, which is important finding for applications of synchrotron radiation in nanoscience.

Using the measured Al x-ray yield obtained with the blank Si sample (see Fig. 3) for which the Al-impurity surface density of $1.8 \times 10^{13}$ atoms $/ \mathrm{cm}^{2}$ was determined with the above-mentioned calibration method, one finds that the detection limit of the high-resolution GEXRF technique, for the standard counting time of $1000 \mathrm{~s}$, is 3.7 $\times 10^{12}$ atoms $/ \mathrm{cm}^{2}$. The corresponding result obtained by Baur et $a l .^{3}$ with the synchrotron-radiation-based lowresolution TXRF technique and for the same counting time is $2.8 \times 10^{10}$ atoms $/ \mathrm{cm}^{2}$. To compare the GEXRF and TXRF results, however, one needs to consider the fact that the TXRF detection limit was obtained for a small angle of incidence, which generally increases the irradiated area on the sample and improves the detection limits. Consequently, by scaling the TXRF result of Baur et $a .^{3}$ by the ratio of the irradiated areas we have found that their detection limits would correspond to about $2 \times 10^{11}$ atoms $/ \mathrm{cm}^{2}$ for a narrow $1 \mathrm{~mm}^{2}$ photon beam spot on the sample. Similarly, the detection limits for $\mathrm{Al}$ of about $5 \times 10^{10}$ atoms $/ \mathrm{cm}^{2}$ reported for conventional TXRF instruments, ${ }^{3}$ which were obtained for irradiated areas of typically tens of $\mathrm{mm}^{2}$, would correspond to $10^{11-12}$ atoms $/ \mathrm{cm}^{2}$ for a $1 \mathrm{~mm}^{2}$ photon beam spot. The present study shows therefore that for Al impurities comparable detection limits can be obtained by using the high-resolution GEXRF and low-resolution TXRF techniques for similar experimental conditions. Furthermore, we would like to emphasize here that the high-resolution GEXRF method combined with the VPD preconcentration technique should permit to push down the detection limit by about 5 orders of magnitude. This results from the fact that the VPD enhancement factor, which for the GEXRF is approximately given by $(D / d)^{2}$ with typical values of $D$ $\approx 300 \mathrm{~mm}$ for the diameter of the Si wafer and $d \approx 1 \mathrm{~mm}$ for the photon beam size on the target, amounts to $10^{5}$. For comparison, the sensitivity improvement for the combined TXRF/VPD technique is expected to be smaller, i.e., 3-4 orders of magnitude, ${ }^{2}$ mainly due to the glancing angle geometry resulting in a larger irradiated sample area. Consequently, the ultimate limit for the detection of Al impurities on $\mathrm{Si}$ wafers by using the combined GEXRF/VPD technique could reach the level of $10^{7}$ atoms $/ \mathrm{cm}^{2}$ which meets the requirements of the Si-based microelectronic technology. An important feature of the described GEXRF technique is that by using narrow synchrotron-radiation beams, in the submicrometer to nanometer range, the lateral and depth profiles of impurities on surfaces can be accessed.

In conclusion, we have shown that the high-resolution GEXRF technique using synchrotron radiation can be used to measure ultralow-level $\mathrm{Al}$ impurities on $\mathrm{Si}$ wafers. The detection limit of the method was estimated to be about $10^{12}$ atoms $/ \mathrm{cm}^{2}$ or even $10^{7}$ atoms $/ \mathrm{cm}^{2}$ if the VPD technique is applied beforehand to the sample. The high sensitivity of the present technique could be achieved thanks to a substantial reduction in the $\mathrm{Si} \mathrm{KL}$ X-ray resonant Raman scattering by optimizing the photon beam energy, which resulted in nearly "background-free" measurement conditions. Finally, the high-resolution GEXRF technique presented here was found to be sensitive to the morphology and chemical state of the surface impurities in the nanometer scale. These properties combined with the lateral and depth profiling capabilities of the method, going ultimately down to the nanometer scale, might be very useful for future applications in nanoscience.

The authors would like to thank the ESRF for support and providing our experiment with very good beam conditions. They also acknowledge the financial support of the Swiss National Science Foundation.
${ }^{1}$ R. Klockenkämper, Total Reflection X-Ray Fluorescence Analysis (Wiley, New York, 1997).

${ }^{2}$ S. Pahlke, Spectrochim. Acta, Part B 58, 2025 (2003).

${ }^{3}$ K. Baur, J. Kerner, S. Brennan, A. Singh, and P. Pianetta, J. Appl. Phys. 88, 4642 (2000).

${ }^{4}$ R. S. Becker, J. A. Golovchenko, and J. R. Patel, Phys. Rev. Lett. 50, 153 (1983).

${ }^{5}$ H. P. Urbach and P. K. de Bokx, Phys. Rev. B 53, 3752 (1996).

${ }^{6}$ A. H. Compton, Philos. Mag. 45, 1121 (1923).

${ }^{7}$ Y. Yoneda and T. Horiuchi, Rev. Sci. Instrum. 42, 1069 (1971).
${ }^{8}$ J. Hoszowska, J.-Cl. Dousse, J. Kern, and Ch. Rhême, Nucl. Instrum. Methods Phys. Res. A 376, 129 (1996).

${ }^{9}$ A. Kotani and S. Shin, Rev. Mod. Phys. 73, 203 (2001).

${ }^{10}$ J. Szlachetko, J.-Cl. Dousse, J. Hoszowska, M. Pajek, R. Barrett, M. Berset, K. Fennane, A. Kubala-Kukuś, and M. Szlachetko, Phys. Rev. Lett. 97, 073001 (2006).

${ }^{11}$ Z. Gai, B. Wu, J. P. Pierce, G. A. Farnan, D. Shu, M. Wang, Z. Zhang, and J. Shen, Phys. Rev. Lett. 89, 235502 (2002).

${ }^{12}$ N. Joshi, A. K. Debnath, D. Aswal, K. P. Muthe, M. S. Kumar, S. K. Gupta, and J. V. Yakhmi, Vacuum 79, 178 (2005). 